\title{
Difficulties Understanding the Explicative Model of Simple DC Circuits in Introductory Physics Courses
}

\author{
Ane Leniz*, Kristina Zuza* and Jenaro Guisasola* \\ *Department of Applied Physics, University of the Basque Country (UPV-EHU), Plaza Europa 1, 20018 \\ Donostia-San Sebastian, Spain
}

\begin{abstract}
Research has shown [1] that understanding the relationship between electrostatics and electrodynamics requires meaningful knowledge about electric concepts. The aim of this investigation is to identify the scope of students' understanding about electric concepts related to the Drude model and the Surface Charge model. In this paper we will describe preliminary results from research at University of the Basque Country (UPV/EHU) and at University of Washington (UW). Some specific examples of the applied questions will be discussed. It will be shown that introductory physics students do not give consistent explanations about the charge movement mechanism on simple DC circuits. The results will be used to develop instructional materials further.
\end{abstract}

Keywords: physics education research, undergraduate, electric potential difference, surface charge density, electric field, understanding

PACS: 01.40.Fk, 41.20.-q

\section{INTRODUCTION}

The model currently taught in most universities and high schools (if not in all) to explain how current works in a de circuit is the Drude model. This model states that the current in a wire is produced by a potential difference between two points of the wire and the electrons are guided by an electric field located inside the conductor with a direction parallel to the wire at any point. One could argue that students have good knowledge and understanding of the simple DC circuit system. However, it has been shown that this does not help to understand the mechanism behind the movement of charges [1]. On the other hand, new studies [2] on ways of teaching direct current circuits propose an approach based on the microscopic mechanisms that produce the charge movement. This approach, called the Surface Charge Model, introduces the mechanism of charge distribution gradient in the circuits' wire surface, giving a different potential at different points of the wire in the circuit. The Surface Charge model enables us to deal with concepts such as electric field and potential difference more exhaustively [3].The SCM model includes a microscopic mechanism to explain the electric current in transient states, such as those introductory physics course students discuss in simple RC circuits and in charging and discharging of capacitors [4].
This paper describes preliminary results from a research at the University of the Basque Country (UPV/EHU) and at the University of Washington (UW). The aim of the investigation is to identify the scope of students' understanding on electric concepts related to the Drude model and the Surface Charge model. Few studies have focused on how students articulate levels describing direct current using both the Drude and the Surface Charge models. Within this context, this work focuses on how students explain phenomena.

\section{RESEARCH CONTEXT}

This research project involved over 110 students at the UPV/EHU in the first year Introductory Physics course for engineers. All of them had taken at least two years of physics in high school and they had passed the national university entrance exams in Spain to study science or engineering. These students took 3.5 hours of lectures and 2 hours of laboratory per week for 14 weeks (second semester) on electromagnetism. Electrostatics and electric circuits were taught for 5 or 6 weeks of this course.

Moreover, the study has also involved 470 students from UW in the E\&M course calculusbased sequence for scientists and engineers. The format of this course (per week) is three 50 minute lectures, one two-hour laboratory session and one 
tutorial section based on Tutorials in Introductory Physics [5] lasting 50 minutes in small groups.

In all cases, lectures were given by experienced teachers of the Physics Department, and the Electricity curriculum in both universities is similar to those of textbooks as for example Tipler and Mosca (2004).

\section{Methodology}

The data was collected through written questions, some of which were online questions. All questions were answered as post-test after receiving instruction.

The same investigator coded all the responses using phenomenographic methodology [6], and recoded them again to ensure consistency between categories (intra-rater reliability Kappa coefficient 0.89). The categories were also discussed with two more investigators who agreed with the exposed code (inter-rater reliability coefficient was 0.85). When there was no agreement, a discussion was held until there was consensus.

Student reasoning was also analyzed to go into greater depth on student understanding [7].

\section{EXAMPLES OF QUESTIONS}

Students were given a variety of questions over the course to investigate their understanding of how current works in a simple dc circuit. The aforementioned questions had different formats, but all were given as post test after receiving instruction. Some of the questions were multiplechoice although a reasoned explanation of the answer was also required. Others were openended questions where students were asked to give an in-depth explanation of the mechanism. The questions probed student understanding of both models in different contexts. A few examples of the aforementioned questions are discussed below.

Students are shown an expanded circuit, which has a short carbon resistor connected by a copper wire to a battery. They are told that it has been connected for a long time and that there is a constant current flowing through it. In addition, they were also told that carbon has much lower conductivity than copper.

Question 1: Students are asked about the electric field at two points of the circuit: one point is located inside the carbon resistor and the other in the copper wire nearby the carbon resistor. Students are supposed to be able to compare the magnitudes of these two electric fields. One correct explanation, according to the Drude model, is that the electric field inside the carbon resistor is larger than in the copper wire, because to maintain constant electric current through the entire circuit the electric field has to be larger in a resistor where conductivity is lower, as the area of the wire is the same throughout the circuit. The surface charge model could also be used to explain that as the surface charge gradient is higher in carbon than in copper, the electric field produced will also be larger in carbon. However, we do not expect students to use this kind of reasoning spontaneously.

Question 2: Students are shown a close-up of a carbon resistor and its surroundings. In this question, students are explicitly asked about the mechanism of current proposed by the surface charge model. They are asked about the change of surface charge density on the carbon and copper surface. One correct explanation is that if we conclude that the electric field inside the carbon resistor is larger, we need a larger change in surface charge density which is producing that larger electric field.

Question 3: Students are asked if the potential difference between two points in the circuit is related to the surface charge density. More specifically, they are asked about the potential difference between a point inside the carbon resistor and another point nearby, in the copper wire. A simple answer could be to argue that there is a relationship between potential difference and surface charge density. As students had already learned in electrostatics, all charge distribution will always be related to the potential generated between two points. The surface charge model has the advantage of explicitly demonstrating this relation.

\section{IDENTIFICATION OF EXPLICATIVE PATTERNS}

The questions described in the previous section do not ask for an answer learned by rote or based on an equation. Students have to analyze the situation and solve it according to an explicative model. In most of the answers students' responses are statements with no justification, or based on wrong learned memory rotes. Other students answer correctly yet justify their response incorrectly.

Despite the curriculum similarities for students at both universities, our aim is not to compare them. In this study we hope to find out about the students' main thinking patterns when they have to interpret concepts such as potential difference, electric field and surface charge density, and see if 
there are similarities in different countries. We discuss the most common tendencies in student explanations, independently of how correct their answer might be.

Regardless of the explanations given for the answer, three main alternative lines of reasoning emerge which are based on a linear or simple causal reasoning. The results obtained in each explanatory category are shown in Table 1.

\section{Charge density variation in the wire implies field and/or potential variation (Category A)}

In category $\mathrm{A}$, all the answers explain that the flow of electricity though the circuit and/or the value of the electric field is proportional to the amount of charge or charge density in the wire. They justify their answer with an explanation based on a causal linear reasoning: there is a chain guiding the potential difference to the electric field, which they relate to the surface charge density. They consider that the potential difference between two points in a circuit is due to difference in charge quantity or density in the wire, but they do not justify it. Explanations like the following were common. "Since the density is equal, the electric field magnitudes will be equal." (question Q1) or "Yes, because the charge distribution affects electric fields which affects the potential difference"(question Q2).

\section{The battery supplies constant current (Category B)}

Answers in category B indicate that the battery supplies a constant current and so, there is no accumulation of charges in the wire. The value of the electric field is proportional to current intensity. Students who use this explanation model consider a macroscopic level of analysis based on $\mathrm{I}=$ constant, in the stationary state. They do not think about the mechanism which makes $\mathrm{I}=$ constant possible, i.e., the surface charge density gradient on the wire. They use a simple causal reasoning to argue that the battery produces an electric current, which is constant, and thus there is no charge accumulation in any part of the circuit, which implies that the surface charge density is constant in the wire. Examples of this kind of answer are the following:

"The electric fields will be the same for both because the current flowing through is constant" (question Q1). "Since the current is constant, the same amount of charge flows through the resistor and the conductor" (question Q2).

\section{Reasoning based on conductivity (Category C)}

Responses in this category explain that the current through the circuit depends on the conductivity of the materials making up the circuit. This explanation states that the material conductivity influences the accumulation of charges in the wire and the value of electric field and potential. This reasoning is based on the conductivity of the circuit's materials. Students use linear causal reasoning which follows this diagram in Q2: a) Carbon has lower conductivity b) and so electrons are less free to move in the carbon, c) this implies smaller (option 1) or larger surface charge density (option 2). Some examples of this category are:

Option 1: "The carbon has lower conductivity so its charges are less free to move around than in copper, so it will become less charged as a result."

Option 2: "The charges flowing through the carbon would slow down and thus become more packed together and dense."

Other students use the same model based on material conductivity but with simple linear reasoning, which is less thorough than the previous model. In Q2, for example, students explain that the carbon's higher resistivity or the copper's higher conductivity produces charge to be accumulated (option 2), or implies less charge in the material (option 1). For example:

Option 1: "Since carbon has lower conductivity than copper, its surface charge density is smaller than the copper wire. There is less positive and negative charges on it." Option 2: If the electrons move slower through the carbon, then they must be squeezed closer together, somewhat like cars in heavy traffic.

It is important to note that, in this question, students are asked about surface charge densities we had expected, they seem unable to identify what they actually are. They tend to use the concept of charges when giving an explanation, and add the adjective "surface" because it is in the statement of the question, without understanding the meaning. 
TABLE 1. Different answer category percentages for the three questions in both UPV $(N=113)$ and UW $(N=476)$

\begin{tabular}{c|c|c|c|c|c|c|c|c}
\hline \multirow{2}{*}{} & \multicolumn{2}{|c|}{ Category A } & \multicolumn{2}{c|}{ Category B } & \multicolumn{2}{c}{ Category C } & \multicolumn{2}{c}{ Other } \\
\cline { 2 - 10 } & UPV/EHU & UW & UPV/EHU & UW & UPV/EHU & UW & UPV/EHU & UW \\
\hline Q1 & $5 \%$ & $37.5 \%$ & $12 \%$ & $9.5 \%$ & $41 \%$ & $7 \%$ & $42 \%$ & $46 \%$ \\
\hline Q2 & - & - & - & $11 \%$ & $42 \%$ & $60 \%$ & $58 \%$ & $29 \%$ \\
\hline Q3 & $24.5 \%$ & $33 \%$ & - & $5 \%$ & $6.5 \%$ & $10 \%$ & $69 \%$ & $52 \%$ \\
\hline
\end{tabular}

However, the vast majority of students are not able to give a justified explanation to any of the questions.

One striking result is the high percentage of incoherently justified or unjustified answers. In the first question (Q1) almost half of them (46\% at UW and $42 \%$ at UPV/EHU) did not coherently explain their answer or did not answer anything at all; in the second question (Q2), these figures stood almost one third of students at UW (29\%) and more than half at UV/EHU (58\%), and in the third question (Q3), more than a half (52\% at UW and $69 \%$ at UPV/EHU). As the questionnaire was part of the final evaluation, and taking into account the way students answered other questions, it does not seem that this result is due to lack of student interest. It may be due to the fact that students do not understand any explicative model to explain the relationship between electric concepts like electric field and potential difference and the movement of charges through the circuit.

\section{DISCUSSION}

Analyzing answers to the questions has revealed that there are three categories of explanations, independently of their country and professor. The model most used by students (category C) consists of supposing that the material conductivity directly influences microscopic properties such as electric field, potential difference and movement of charges. This overgeneralization leads students to failure when they analyze questions involving the value of field and potential difference in a circuit.

Another result is the difficulty that students have recognizing the surface charges on the wire. Students speak about the flow of charges into the wire (category A). One possible explanation is that most typical textbooks propose a model which only mentions the current flow into the wire but not the mechanism that produces the current. So students are not used to thinking about how surface charge density gradients affect the current.

The results show that the majority of students do not give consistent explanations to the charge movement mechanism on simple DC circuits. A minority of answers explain in a scientific way the three questions (about $10 \%$ at both universities). Students' explicative models are mostly poor and the vast majority do not use not the Drude model neither any microscopic mechanism that explains the electric current in stationary states.

The results have major implications on the instruction. It is necessary to carry out additional research to identify new instructional strategies which would help students' conceptual understanding, and develop better explicative models that should link their electrostatics comprehension to the study of simple DC circuits.

\section{ACKNOWLEDGMENTS}

The authors would like to thank Paula Heron and Peter Shaffer for the discussion about the questions, and also Ryan Hazelton and all the members of the Physics Education Research Group at University of Washington who facilitated this research.

\section{REFERENCES}

1. J. Guisasola, Teaching and learning Electricity: The relations between macroscopic level observation and micrsocopic level theories. In M.R. Metthews (ed.) International Handbook of Research in History, Phylosophy and Science Teaching, (Springer, Drodrecht, 2014), Chap. 5, pp. 129-156.

2. J. Guisasola, J. L. Zubimendi and K. Zuza, Phys. Rev. ST-PER 6, 020102 (2010).

3. H. Haertel, (private communication, 2012)

4. R. W. Chabay, and B. A. Sherwood, Matter and Interactions, $3^{\text {rd }}$ ed, (Wiley, New York, 2011).

5. R. L. C. Hazelton, M. R. Stetzer, P. R. L. Heron and P S. Shaffer, AIP Conf. Proc. 1513, 166 (2013).

6. L. C. McDermott, P. S. Shaffer, and the P.E.G. at the U.W., Tutorials in Introductory Physics (Pearson Learning Solutions, 2011).

7. F. Marton, Instructional Science 10, 177-200 (1981).

8. F. Halbwachs, Eur. J. of Sci. Edu. 1(2), 169-171 (1979). 